\title{
Anomalous coupling studies at the LHC with proton tagging
}

\author{
Christophe Royon ${ }^{1, a}$ and Matthias Saimpert ${ }^{1, b}$ \\ ${ }^{1}$ DSM/IRFU/SPP, CEA Saclay, F91 191 Gif-sur-Yvette, France
}

\begin{abstract}
We present the photon induced processes leading to intact protons at the final state that can be studied at the LHC. We give the cross section predicted by the standard model for $W$ and photon pair production. The study of these processes lead to unprecedented sensitivities on quartic anomalous couplings between photons and $W, Z$ bosons, reaching the values expected for extra dimension models.
\end{abstract}

In this short report, we discuss some potential measurements to be performed using proton tagging detectors at the LHC. The main motivation is to explore rare events, searching for beyond standard model physics such as quartic anomalous couplings between photons and $W / Z$ bosons and photons. We assume in the following intact protons to be tagged in dedicated detectors located at about $210 \mathrm{~m}$ for ATLAS (220 m for CMS) as described in Ref. [1].

In the first part of this report, we discuss the Standard Model (SM) production of $W$ and $\gamma$ pairs at the LHC via photon exchanges. In the second, third and fourth sections, we discuss the sensitivities of these processes to quartic gauge anomalous couplings.

\section{Standard Model exclusive $\gamma \gamma, W W$ and ZZ production}

\subsection{Standard Model exclusive $\gamma \gamma$ production at the LHC: Photon and gluon induced processes}

In Fig. 1 and 2, we show the leading processes producing two photons and two intact protons in the final state as an example. The first diagram (Fig. 1) corresponds to exclusive QCD di-photon production via gluon exchanges (the second gluon ensures that the exchange is colorless leading to intact protons in the final state) and the second one (Fig. 2) via photon exchanges, It is worth noticing that quark, lepton and $W$ loops need to be considered in order to get the correct SM cross section for di-photon production, as shown in Fig 2. The QCD induced processes from the Khoze Martin Ryskin model are dominant at low masses whereas the photon induced ones (QED processes) dominate at higher di-photon masses [2]. It is very important to notice that the $W$ loop contribution dominates at high di-photon masses [3-5] whereas this contribution is omitted in most studies. We included those processes in the Forward Physics Monte Carlo generator (FPMC) [6],

\footnotetext{
a e-mail: christophe.royon@cea.fr

be-mail: matthias.saimpert@cea.fr
}

which is as far as we know the first MC to include all of them in a single framework.

\subsection{Standard Model $W W$ and $Z Z$ production}

In the standard model of particle physics, the couplings of fermions and gauge bosons are constrained by the gauge symmetries of the Lagrangian. The measurement of $W$ and $Z$ boson pair productions via the exchange of two photons allows to provide directly stringent tests of one of the most important and least understood mechanism in particle physics, namely the electroweak symmetry breaking. In the pure $\mathrm{SM}, \mathrm{ZZ}$ production is forbidden and its observation would be a direct sign of new physics.

The process that we study is the $W / Z$ pair production induced by the exchange of two photons [7]. It is a pure QED process in which the decay products of the $W / Z$ bosons are measured in the central detector and the scattered protons leave intact in the beam pipe at very small angles and are detected in AFP or CT-PPS. All these processes as well as the different diffractive backgrounds were implemented in the FPMC Monte Carlo [6].

After simple cuts to select exclusive $W$ pairs decaying into leptons, such as a cut on the proton momentum loss of the proton $(0.0015<\xi<0.15)$ - which represent the acceptance of the AFP or CT-PPS detectors at 210 and $420 \mathrm{~m}$ - on the transverse momentum of the leading and second leading leptons at 25 and $10 \mathrm{GeV}$ respectively, on $\mathscr{E}_{T}>20 \mathrm{GeV}, \Delta \phi>2.7$ between leading leptons, and $160<W<500 \mathrm{GeV}, W$ being the diffractive mass reconstructed using the forward detectors, the background is found to be less than 1.7 event for $30 \mathrm{fb}^{-1}$ for a SM signal of 51 events [7].

\section{Quartic $W W$ and $Z Z$ anomalous couplings}

In this section, we discuss the quartic $\gamma \gamma W W$ and $\gamma \gamma Z Z$ anomalous couplings (the trilinear couplings were discussed in Ref. [8]). The parameterization of the quartic 


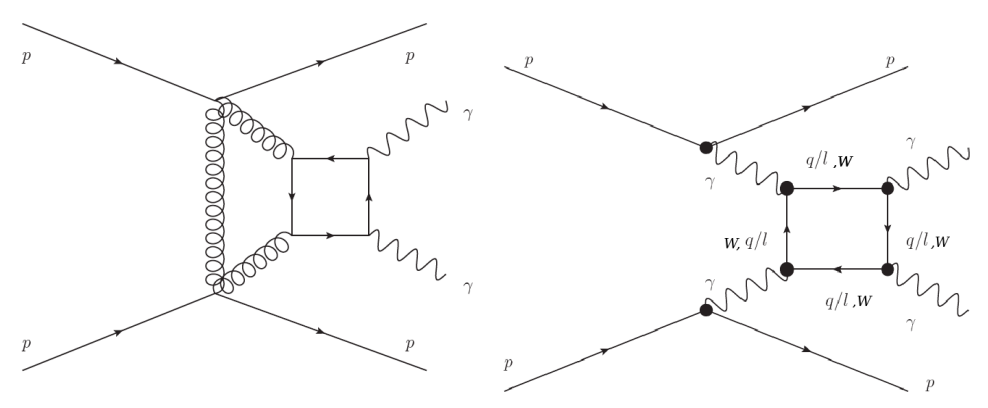

Figure 1. di-photon QCD exclusive production (left) and di-photon production via photon exchanges (right) at the lowest order of perturbation theory.

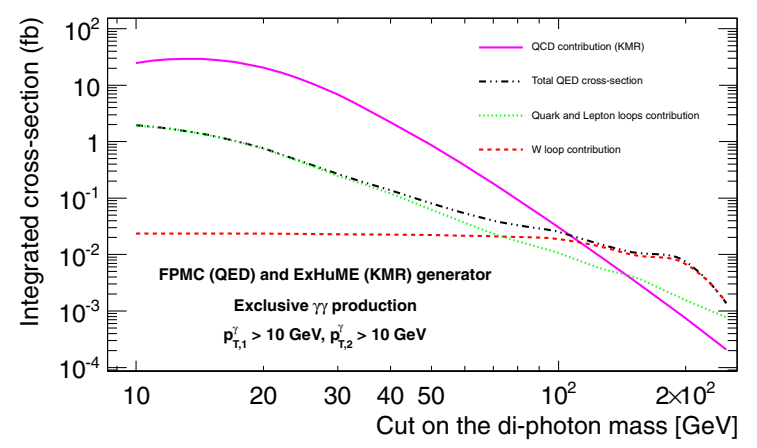

Figure 2. Integrated cross sections of the different exclusive di-photon processes with intact protons at the 13 TeV LHC, plotted against the required minimum di-photon mass. Both photons are required to have a transverse momentum above $10 \mathrm{GeV}$.

couplings developped in Ref. [9] is adopted. The cuts to select quartic anomalous gauge couplings $W W$ events are similar as the ones we mentioned in the previous section, namely $0.0015<\xi<0.15$ for the tagged protons corresponding to the AFP or CT-PPS detector at 210 and $420 \mathrm{~m}$, $\mathbb{E}_{T}>20 \mathrm{GeV}, \Delta \phi<3.13$ between the two leptons. In addition, a cut on the $p_{T}$ of the leading lepton $p_{T}>160 \mathrm{GeV}$ and on the diffractive mass $W>800 \mathrm{GeV}$ are requested since anomalous coupling events appear at high mass. After these requirements, we expect about 0.7 background events for an expected signal of 17 events if the anomalous couplings is about four orders of magnitude lower than the present LEP limit [10] $\left(\left|a_{0}^{W} / \Lambda^{2}\right|=5.410^{-6}\right)$ or two orders of magnitude lower with respect to the D0 and CDF limits [11] for a luminosity of $30 \mathrm{fb}^{-1}$. The strategy to select anomalous couplings $Z Z$ events is analogous and the presence of three leptons or two same sign leptons are requested. Table 1 gives the reach on anomalous couplings at the LHC for luminosities of 30 and $200 \mathrm{fb}^{-1}$ compared to the present OPAL limits from the LEP accelerator [10]. More recent limits were published recently by the D0 and CMS collaborations [11] on $a_{0}^{W}$ and $a_{C}^{W}$, and they are respectively $1.510^{-4}$ and $510^{-4}$ from CMS with a form factor of $500 \mathrm{GeV}$. D0 limits are lower.

With the new predicted sensitivity it is possible to reach the values expected in extra dimension models. The tagging of the protons using the ATLAS Forward Physics detectors is the only method at present to test so small values of quartic anomalous couplings.
The search for quartic anomalous couplings between $\gamma$ and $W$ bosons was performed again after a full simulation of the ATLAS detector including pile up [12] assuming the protons to be tagged in AFP or CT-PPS [1] at $210 \mathrm{~m}$ only. Integrated luminosities of 40 and $300 \mathrm{fb}^{-1}$ with, respectively, 23 or 46 average pile up events per beam crossing have been considered. In order to reduce the background, each $W$ is assumed to decay leptonically (note that the semi-leptonic case in under study). The full list of background processes used for the ATLAS measurement of Standard Model $W W$ cross-section was simulated, namely $t \bar{t}, W W, W Z, Z Z, W+$ jets, Drell-Yan and single top events. In addition, the additional diffractive backgrounds mentioned in the previous paragraph were also simulated, The requirement of the presence of at least one proton on each side of AFP or CT-PPS within a time window of 10 ps allows us to reduce the background by a factor of about 200 (50) for $\mu=23$ (46). The $p_{T}$ of the leading lepton originating from the leptonic decay of the $W$ bosons is required to be $p_{T}>150 \mathrm{GeV}$, and that of the next-to-leading lepton $p_{T}>20 \mathrm{GeV}$. Additional requirement of the dilepton mass to be above $300 \mathrm{GeV}$ allows us to remove most of the di-boson events. Since only leptonic decays of the $\mathrm{W}$ bosons are considered, we require in addition less than 3 tracks associated to the primary vertex, which allows us to reject a large fraction of the non-diffractive backgrounds (e.g. $t \bar{t}$, di-boson productions, $W+$ jet, etc.) since they show much higher track multiplicities. Remaining Drell-Yan and QED backgrounds are suppressed by re- 
Table 1. Reach on anomalous couplings obtained in $\gamma$ induced processes after tagging the protons in AFP or CT-PPS compared to the present OPAL limits. The $5 \sigma$ discovery and $95 \%$ C.L. limits are given for a luminosity of 30 and $200 \mathrm{fb}^{-1}$ [7]

\begin{tabular}{|c||c|c|c|}
\hline \multicolumn{1}{|c|}{ Couplings } & OPAL limits & \multicolumn{3}{|c|}{ Sensitivity @ $\mathcal{L}=30(200) \mathrm{fb}^{-1}$} \\
& {$\left[\mathrm{GeV}^{-2}\right]$} & $5 \sigma$ & $95 \% \mathrm{CL}$ \\
\hline$a_{0}^{W} / \Lambda^{2}$ & {$[-0.020,0.020]$} & $5.410^{-6}$ & $2.610^{-6}$ \\
& & $\left(2.710^{-6}\right)$ & $\left(1.410^{-6}\right)$ \\
\hline$a_{C}^{W} / \Lambda^{2}$ & {$[-0.052,0.037]$} & $2.010^{-5}$ & $9.410^{-6}$ \\
& & $\left(9.610^{-6}\right)$ & $\left(5.210^{-6}\right)$ \\
\hline$a_{0}^{Z} / \Lambda^{2}$ & {$[-0.007,0.023]$} & $1.410^{-5}$ & $6.410^{-6}$ \\
& & $\left(5.510^{-6}\right)$ & $\left(2.510^{-6}\right)$ \\
\hline$a_{C}^{Z} / \Lambda^{2}$ & {$[-0.029,0.029]$} & $5.210^{-5}$ & $2.410^{-5}$ \\
& & $\left(2.010^{-5}\right)$ & $\left(9.210^{-6}\right)$ \\
\hline \hline
\end{tabular}

quiring the difference in azimuthal angle between the two leptons $\Delta \phi<3.1$. After these requirements, a similar sensitivity with respect to fast simulation without pile up was obtained.

\section{Quartic photon anomalous couplings}

\subsection{Theretical motivations}

In this section, four-photon $(4 \gamma)$ interactions through diphoton production via photon fusion with intact outgoing protons are considered. In the assumption of a new physics mass scale $\Lambda$ heavier than experimentally accessible energy $E$, all new physics manifestations can be described using an effective Lagrangian valid for $\Lambda \gg E$. Among these operators, the pure photon dimension-eight operators

$$
\mathcal{L}_{4 \gamma}=\zeta_{1}^{\gamma} F_{\mu \nu} F^{\mu \nu} F_{\rho \sigma} F^{\rho \sigma}+\zeta_{2}^{\gamma} F_{\mu \nu} F^{v \rho} F_{\rho \lambda} F^{\lambda \mu}
$$

can induce the $\gamma \gamma \gamma \gamma$ process, highly suppressed in the $\mathrm{SM}[3,13]$. We discuss here possible new physics contributions to $\zeta_{1,2}^{\gamma}$ that can be probed and discovered at the LHC using the forward proton detectors.

Loops of heavy charged particles contribute to the $4 \gamma$ couplings [3,13] as $\zeta_{i}^{\gamma}=\alpha_{\mathrm{em}}^{2} Q^{4} m^{-4} N c_{i, s}$, where $c_{1, s}$ is related to the spin of the heavy particle of mass $m$ running in the loop and $Q$ its electric charge. The factor $N$ counts all additional multiplicities such as color or flavor. These couplings scale as $\sim Q^{4}$ and are enhanced in presence of particles with large charges. For example, certain light composite fermions, characteristic of composite Higgs models, have typically electric charges of several units. For a $500 \mathrm{GeV}$ vector (fermion) resonance with $Q=3$ (4), large couplings $\zeta_{i}^{\gamma}$ of the order of $10^{-13}-10^{-14}$ $\mathrm{GeV}^{-4}$ can be reached.

Beyond perturbative contributions to $\zeta_{i}^{\gamma}$ from charged particles, non-renormalizable interactions of neutral particles are also present in common extensions of the SM. Such theories can contain scalar, pseudo-scalar and spin2 resonances that couple to the photon and generate the $4 \gamma$ couplings by tree-level exchange as $\zeta_{i}^{\gamma}=\left(f_{s} m\right)^{-2} d_{i, s}$, where $d_{1, s}$ is related to the spin of the particle. Stronglycoupled conformal extensions of the SM contain a scalar particle $\left(s=0^{+}\right)$, the dilaton. Even a $2 \mathrm{TeV}$ dilaton can produce a sizable effective photon interaction, $\zeta_{1}^{\gamma} \sim 10^{-13}$ $\mathrm{GeV}^{-4}$. These features are reproduced at large number of colors by the gauge-gravity correspondence in a warped extra dimension. Warped-extra dimensions also feature Kaluza-Klein (KK) gravitons [14], that can induce anomalous couplings [13]

$$
\zeta_{i}^{\gamma}=\frac{\kappa^{2}}{8 \tilde{k}^{4}} d_{i, 2}
$$

where $\tilde{k}$ is the IR scale that determines the first KK graviton mass and $\kappa$ is a parameter that can be taken $O(1)$. For $\kappa \sim 1$, and $m_{2} \lesssim 6 \mathrm{TeV}$, the photon vertex can easily exceed $\zeta_{2}^{\gamma} \sim 10^{-14} \mathrm{GeV}^{-4}$.

\subsection{Experimental sensitivity to quartic four photon couplings}

As we mentionned already, the $\gamma \gamma \gamma \gamma$ process (Fig. 2) can be probed via the detection of two intact protons in the forward proton detectors and two energetic photons in the corresponding electromagnetic calorimeters. The SM cross section of di-photon production with intact protons is dominated by the QED process at high di-photon mass — and not by gluon exchanges - and is thus very well known.

As mentioned in Ref. [15], the photon identification efficiency is expected to be around $75 \%$ for $p_{T}>100 \mathrm{GeV}$, with jet rejection factors exceeding 4000 even at high pile up (>100). In addition, about $1 \%$ of the electrons are misidentified as photons. These numbers are used in the phenomenological study presented below.

As for the previous studies, the anomalous $\gamma \gamma \gamma \gamma$ process has been implemented in the Forward Physics Monte Carlo (FPMC) generator [6]. The FPMC generator was also used to simulate the background processes giving rise to two intact protons accompanied by two photons, electrons or jets that can mimic the photon signal. Those include exclusive SM production of $\gamma \gamma \gamma \gamma$ via lepton and quark boxes and $\gamma \gamma \rightarrow e^{+} e^{-}$. The central exclusive production of $\gamma \gamma$ via two-gluon exchange, not present in FPMC, was simulated using ExHuME [16]. This series of backgrounds is called "Exclusive" in Table 2 and Fig. 3, 4. 


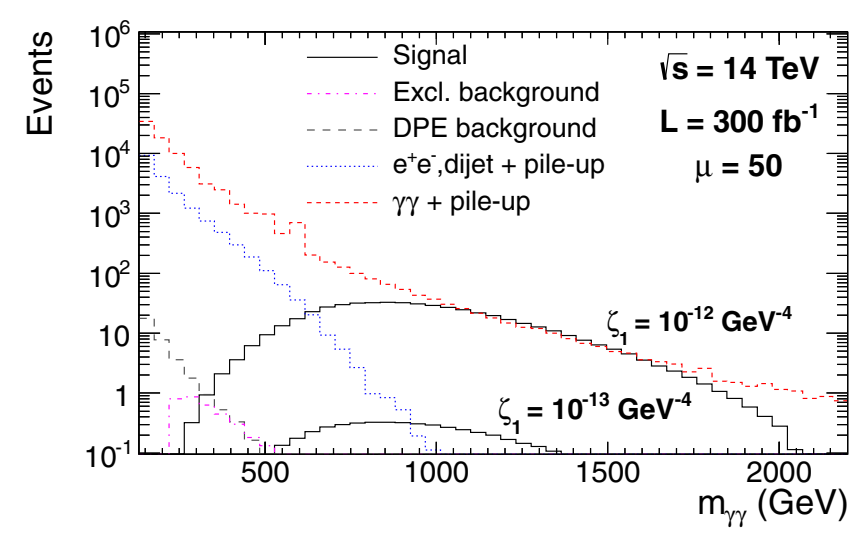

Figure 3. Di-photon invariant mass distribution for the signal $\left(\zeta_{1}=10^{-12}, 10^{-13} \mathrm{Gev}^{-4}\right.$, see Eq. 1) and for the backgrounds (dominated by $\gamma \gamma$ with protons from pile up), requesting two protons in the forward detectors and two photons of $p_{T}>50 \mathrm{GeV}$ with at least one converted photon in the central detector, for a luminosity of $300 \mathrm{fb}^{-1}$ and an average pile up of $\mu=50$.

Table 2. Number of signal (for a baseline coupling of $\mathrm{GeV}^{-4}$ ) and background events after various selections for an integrated luminosity of $300 \mathrm{fb}^{-1}$ and $\mu=50$ at $\sqrt{s}=14 \mathrm{TeV}$. At least one converted photon is required. The standard cuts correspond to the AFP or CT-PPS acceptance $(0.015<\xi<0.15)$ and the request of the photon $p_{T}$ to be above $50 \mathrm{GeV}$.

\begin{tabular}{|c||c||c|c|c|c|}
\hline Cut / Process & Signal & Excl. & DPE & $\begin{array}{c}\mathrm{e}^{+} \mathrm{e}^{-} \text {, dijet } \\
+ \text { pile up }\end{array}$ & $\begin{array}{c}\gamma \gamma+ \\
\text { pile-up }\end{array}$ \\
\hline \hline standard & 20.8 & 3.7 & 48.2 & $2.8 \cdot 10^{4}$ & $1.0 \cdot 10^{5}$ \\
$p_{\mathrm{T} 1}>200 \mathrm{GeV}, p_{\mathrm{T} 2}>100 \mathrm{GeV}$ & 17.6 & 0.2 & 0.2 & 1.6 & 2968 \\
$m_{\gamma \gamma}>600 \mathrm{GeV}$ & 16.6 & 0.1 & 0 & 0.2 & 1023 \\
$p_{\mathrm{T} 2} / p_{\mathrm{T} 1}>0.95,|\Delta \phi|>\pi-0.01$ & 16.2 & 0.1 & 0 & 0 & 80.2 \\
$\sqrt{\xi_{1} \xi_{2} s}=m_{\gamma \gamma} \pm 3 \%$ & 15.7 & 0.1 & 0 & 0 & 2.8 \\
$\left|y_{\gamma \gamma}-y_{p p}\right|<0.03$ & 15.1 & 0.1 & 0 & 0 & 0 \\
\hline
\end{tabular}

FPMC was also used to produce $\gamma \gamma$, Higgs to $\gamma \gamma$ and dijet productions via double pomeron exchange (called DPE background in Table 2 and Fig. 3). Such backgrounds tend to be softer than the signal and can be suppressed with requirements on the transverse momenta of the photons and the di-photon invariant mass. In addition, the final-state photons of the signal are typically back-to-back and have about the same transverse momenta. Requiring a large azimuthal angle $|\Delta \phi|>\pi-0.01$ between the two photons and a ratio $p_{T, 2} / p_{T, 1}>0.95$ greatly reduces the contribution of non-exclusive processes.

Additional background processes include the quark and gluon-initiated production of two photons, two jets and Drell-Yan processes leading to two electrons. The two intact protons arise from pile up interactions (these backgrounds are called $\gamma \gamma+$ pile up and $\mathrm{e}^{+} \mathrm{e}^{-}$, dijet + pileup in Table 2). These events were produced using HERWIG [17] and PYTHIA [18]. The pile up background is further suppressed by requiring the proton missing invariant mass to match the di-photon invariant mass within the expected resolution and the di-photon system rapidity and the rapidity of the two protons to be similar.

The number of expected signal and background events passing respective selections is shown in Table 2 for an integrated luminosity of $300 \mathrm{fb}^{-1}$ and for a center-of-mass energy of $14 \mathrm{TeV}$. Exploiting the full event kinematics with the forward proton detectors allows to completely suppress the background with a signal selection efficiency after the acceptance cuts exceeding $70 \%$. Tagging the protons is absolutely needed to suppress the $\gamma \gamma+$ pile up events. Further background reduction is even possible by requiring the photons and the protons to originate from the same vertex that provides an additional rejection factor of 40 for 50 pile up interactions, showing the large margin on the background suppression. A similar study at a higher pile up of 200 was performed and led to a very small background. The sensitivities on photon quartic anomalous couplings are given in Table 3 . The sensitivity extends up to $7 \cdot 10^{-15}$ $\mathrm{GeV}^{-4}$, allowing us to probe further the models of new physics described above.

If discovered at the LHC, $\gamma \gamma \gamma \gamma$ quartic anomalous couplings would be a major discovery which could be related to the existence of extra dimensions in the universe. In addition, it might be inveestigated if there could be a link with some experiments in atomic physics. As an example, the Aspect photon correlation experiments [19] might be interpreted via the existence of extra dimensions. Photons could communicate through extra dimensions and the deterministic interpretation of Einstein for these experiments might be true if such anomalous couplings exist. From the point of view of atomic physics, the results of the Aspect experiments would depend on the distance of 

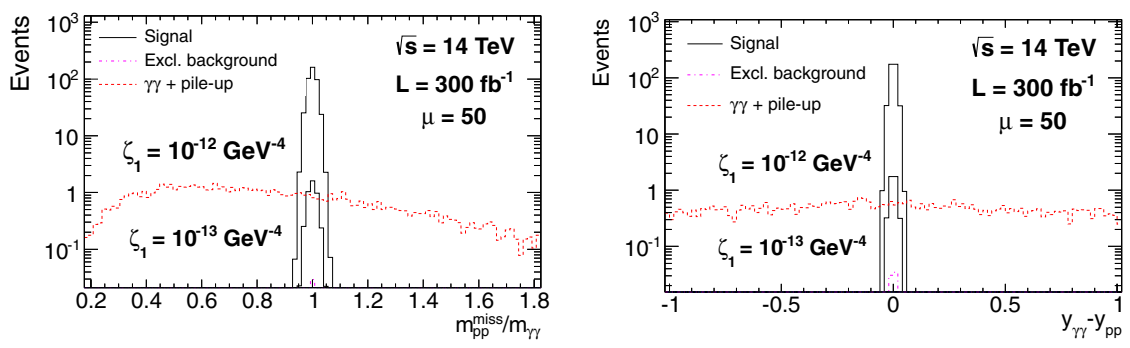

Figure 4. Di-photon to missing proton mass ratio (left) and rapidity difference (right) distributions for signal considering two different coupling values $\left(10^{-12}\right.$ and $10^{-13} \mathrm{GeV}^{-4}$, see Eq. 1) and for backgrounds after requirements on photon $p_{T}$, di-photon invariant mass, $p_{T}$ ratio between the two photons and on the angle between the two photons. At least one converted photon is required. The integrated luminosity is $300 \mathrm{fb}^{-1}$ and the average pile up is $\mu=50$.

Table 3. $5 \sigma$ discovery and $95 \% \mathrm{CL}$ exclusion limits on $\zeta_{1}$ and $\zeta_{2}$ couplings in $\mathrm{GeV}^{-4}$ (see Eq. 1) with and without form factor (f.f.) with $\Lambda^{\prime}=1 \mathrm{TeV}$, requesting at least one converted photon ( $\geq 1$ conv. $\gamma$ ) or not (all $\gamma$ ). All sensitivities are given for $300 \mathrm{fb}^{-1}$ and $\mu=50$ pile up events (medium luminosity LHC) except for the numbers of the last column which are given for $3000 \mathrm{fb}^{-1}$ and $\mu=200$ pile up events (high luminosity LHC) where we do not request converted photons in the case of ATLAS.

\begin{tabular}{|c||c|c||c||c|}
\hline Luminosity & $300 \mathrm{fb}^{-1}$ & $300 \mathrm{fb}^{-1}$ & $300 \mathrm{fb}^{-1}$ & $3000 \mathrm{fb}^{-1}$ \\
\hline pile up $(\mu)$ & 50 & 50 & 50 & 200 \\
\hline \hline coupling & $\geq 1$ conv. $\gamma$ & $\geq 1$ conv. $\gamma$ & all $\gamma$ & all $\gamma$ \\
$\left(\mathrm{GeV}^{-4}\right)$ & $5 \sigma$ & $95 \% \mathrm{CL}$ & $95 \% \mathrm{CL}$ & $95 \% \mathrm{CL}$ \\
\hline$\zeta_{1}$ f.f. & $1 . \cdot 10^{-13}$ & $9 . \cdot 10^{-14}$ & $5 . \cdot 10^{-14}$ & $2.5 \cdot 10^{-14}$ \\
$\zeta_{1}$ no f.f. & $3.5 \cdot 10^{-14}$ & $2.5 \cdot 10^{-14}$ & $1.5 \cdot 10^{-14}$ & $7 . \cdot 10^{-15}$ \\
\hline$\zeta_{2}$ f.f. & $2.5 \cdot 10^{-13}$ & $1.5 \cdot 10^{-13}$ & $1 . \cdot 10^{-13}$ & $4.5 \cdot 10^{-14}$ \\
$\zeta_{2}$ no f.f. & $7.5 \cdot 10^{-14}$ & $5.5 \cdot 10^{-14}$ & $3 . \cdot 10^{-14}$ & $1.5 \cdot 10^{-14}$ \\
\hline
\end{tabular}

the two photon sources. Furthermore, extra dimensions might be relevant also for the fast expansion of the universe within inflation models.

\section{Conclusion}

In this short report, we detailled the interest of tagging the intact protons to study in detail $W W, Z Z$ and $\gamma \gamma$ productions via photon exchanges. Uprecedented sensitivities can be achieved at the LHC in the CMS-TOTEM and ATLAS experiments on quartic anomalous gauge couplings, especially on $\gamma \gamma \gamma \gamma$ couplings, which lead to one of the best sensitivity on extra dimensions at the LHC.

\section{Acknowledgments}

These results come from a fruitful collaboration with Emilien Chapon, Sylvain Fichet, Gero von Gersdorff, Oldřich Kepka, Bruno Lenzi.

\section{References}

[1] N. Cartiglia, C. Royon, Int J Mod Phys, A special issue on DPE/CEP, Editors: M. Albrow, V. Khoze, C. Royon.

[2] V. A. Khoze, A. D. Martin and M. G. Ryskin, Eur. Phys. J. C 23 (2002) 311.
[3] S. Fichet, G. von Gersdorff, O. Kepka, B. Lenzi, C. Royon, M. Saimpert, Phys. Rev. D89 (2014) 114004.

[4] S. Fichet, G. von Gehrsdorff, O. Kepka, C. Royon, M. Saimpert, arXiv:1411.6629.

[5] D. d'Enterria, G. da Silveira, Phys. Rev. Lett. 111 (2013) 080405; H. Sun, Eur.Phys.J. C74 (2014) 2977.

[6] M. Boonekamp, A. Dechambre, V. Juranek, O. Kepka, M. Rangel, C. Royon, R. Staszewski, ePrint: arXiv:1102.2531; M. Boonekamp, V. Juranek, O. Kepka, C. Royon "Forward Physics Monte Carlo", "Proceedings of the workshop: HERA and the LHC workshop series on the implications of HERA for LHC physics," arXiv:0903.3861 [hep-ph].

[7] E. Chapon, O. Kepka, C. Royon, Phys. Rev. D81 (2010) 074003; J. de. Favereau et al., preprint arXiv:0908.2020.

[8] O. Kepka and C. Royon, Phys. Rev. D 78 (2008) 073005.

[9] G. Belanger and F. Boudjema, Phys. Lett. B 288 (1992) 201.

[10] G. Abbiendi et al. [OPAL Collaboration], Phys. Rev. D 70 (2004) 032005 [arXiv:hep-ex/0402021].

[11] CMS Coll., JHEP 07 (2013) 116; D0. Coll., Phys. Rev. D 88 (2013) 012005.

[12] ATLAS Coll., CERN-LHCC-2011-012; M.Albrow et al., CMS-TOTEM Precision Proton Spectrometer, CERN-LHCC-2014-021. 
[13] S. Fichet and G. von Gersdorff, preprint arXiv:1311.6815.; R. S. Gupta, Phys. Rev. D 85 (2012) 014006.

[14] L. Randall and R. Sundrum, Phys. Rev. Lett. 83 (1999) 3370.

[15] ATLAS Coll., ATL-PHYS-PUB-2013-009; ATLAS Coll., JINST, Vol. 3 (2008) S08003.

[16] J. Monk and A. Pilkington, Comput. Phys. Commun. 175 (2006) 232; V.A. Khoze, A.D. Martin, M.G.
Ryskin, Eur.Phys.J. C 55 (2008) 363.

[17] G. Corcella et al., arXiv:hep-ph/0210213.

[18] T. Sjostrand, S. Mrenna and P. Z. Skands, Comput. Phys. Commun. 178 (2008) 852.

[19] A. Aspect, P. Grangier, G. Roger, Phys. Rev. Lett., Vol. 49, no 2 (1982) 91-94; A. Aspect, J. Dalibard, G. Roger, Phys. Rev. Lett., Vol. 49, Iss. 25 (1982) 1804. 